\title{
Global linear stability of the boundary-layer flow over a rotating sphere
}

\author{
A. Barrow ${ }^{\mathrm{a}}$, S.J. Garrett ${ }^{*, a}$, N. Peake ${ }^{\mathrm{b}}$ \\ ${ }^{a}$ Department of Mathematics, University of Leicester, Leicester, LE1 7RH, UK \\ ${ }^{b}$ DAMTP, Centre for Mathematical Sciences, University of Cambridge, \\ Wilberforce Road, Cambridge, CB3 OWA, UK
}

\begin{abstract}
We consider the linear global stability of the boundary-layer flow over a rotating sphere. Our results suggest that a self-excited linear global mode can exist when the sphere rotates sufficiently fast, with properties fixed by the flow at latitudes between approximately $55^{\circ}-65^{\circ}$ from the pole (depending on the rotation rate). A neutral curve for global linear instabilities is presented with critical Reynolds number consistent with existing experimentally measured values for the appearance of turbulence. The existence of an unstable linear global mode is in contrast to the literature on the rotating disk, where it is expected that nonlinearity is required to prompt the transition to turbulence. Despite both being susceptible to local absolute instabilities, we conclude that the transition mechanism for the rotating-sphere flow may be different to that for the rotating disk.
\end{abstract}

Key words: Boundary-layer flow, rotating sphere, global modes

\footnotetext{
${ }^{*}$ Corresponding author

Email address: stephen.garrett@le.ac.uk (S.J. Garrett)

$U R L$ : www.tinyurl.com/SJGarrett (S.J. Garrett)
} 


\section{Introduction}

The stability of the boundary layer on rotating bodies of revolution has received considerable attention over a number of years. A significant advance has been made by Lingwood [1], who showed that the boundary layer on a rotating disk of infinite extent is locally absolutely unstable at Reynolds numbers in excess of a critical value (equivalent to being outside a critical radius at fixed rotation rate), and is at worst convectively unstable inside this radius. The value of the critical Reynolds number agrees exceedingly well with experimentally measured values of the transition Reynolds number, leading to Lingwood's hypothesis that absolute instability plays a rôle in turbulent transition on the rotating disk. Lingwood [2] later experimentally confirmed the presence of absolute instability above a fixed, critical Reynolds number very close to the theoretical value by tracking the wavepacket response to an impulse excitation on a rotating disk; thereby adding weight to her initial assertion.

A few years later, Davies \& Carpenter [3] performed direct numerical simulations solving the linearized Navier-Stokes equations directly on a rotating disk of infinite extent. When they made the same homogeneous flow approximation as in Lingwood's analysis, they recovered her results in full, with absolute instability clearly present at high Reynolds number. However, when the spatial inhomogeneity of the boundary layer was included there was no evidence that the local absolute instability gives rise to the unstable global oscillator in the long-time response that would be required to suggest the onset of transition within a purely linear theory. Indeed their study suggests that convective behaviour eventually dominates at all the Reynolds 
numbers investigated, even for strongly locally absolutely unstable regions. Their conclusion was that absolute instability was not involved in the transition process through linear effects. These theoretical results have since been supported experimentally by Othman \& Corke [4].

Following this, Pier [5] demonstrated explicitly that a nonlinear approach is required to explain the self-sustained behaviour of the rotating-disk flow. Using the result of Huerre \& Monkewitz [6] that the presence of local absolute instability does not necessarily give rise to linear global instability, Pier suggested that the flow has a primary nonlinear global mode (fixed by the onset of the local absolute instability) which has a secondary absolute instability that triggers the transition to turbulence. Some experimental evidence for a secondary instability exists [11-13], but its behaviour and relation to the primary absolute instability are not fully understood as yet, although some considerable advances in related geometries have been made recently by Viaud et al. [14, 15].

In an attempt to explain Lingwood's [2] original experimental observations in the light of the subsequent theoretical developments, Healey [16] presented a theory, based on the Ginzburg-Landau equation, that suggests that there can be a linear global instability when there is local absolute instability in a finite domain (thereby representing the edge of the disk). The finite size of experimental disks is of course a crucial difference between experimental and theoretical studies prior to this work. Healey demonstrated that, under particular assumptions about the flow at the edge of the disk, the effects of finite size are destabilizing in a linear setting but stabilizing in the nonlinear setting. Contrary to Healey's theoretical study, the experimen- 
tal study of Imayama et al. [17] found no clear experimental evidence that edge effects would either enhance or reduce stability. Pier [18] has recently suggested that this apparent discrepancy could be reconciled with a change in Healey's assumptions for the flow at the edge of the disk.

A theory of transition over the rotating disk continues to develop and, with the new developments around edge effects in mind, we feel it instructive to present our study of the linear global instability of the boundary-layer flow over a rotating sphere. The spherical geometry is such that theoretical studies do not suffer from edge effects in the same way, although of course the colliding boundary layers orginating from the two poles must erupt at the equator, presenting a barrier to boundary-layer stability calculations. In this study we consider the linear global modes of the rotating-sphere system, as formulated for weakly nonparallel shear flow by Monkewitz et al. [19]. The idea is to use data from the local absolute instability analysis of Garrett \& Peake [20] and Barrow \& Garrett [21] to construct solutions for the entire flow with single complex frequency $\gamma_{G}$. The long-time response of the system is then governed by $\operatorname{Im}\left(\gamma_{G}\right)$, and will be linearly globally stable if $\operatorname{Im}\left(\gamma_{G}\right)<0$ and globally unstable if $\operatorname{Im}\left(\gamma_{G}\right)>0$. The approach in this paper is to attempt to determine $\gamma_{G}$ for the rotating-sphere flow.

As discussed by Garrett [20, 22], the rotating-sphere flow has a number of similarities with that over a rotating disk. In particular, for sufficiently high rotation rate the flow exhibits the same distinct flow regimes (laminar, transitional, turbulent) and these move towards the pole with further increases in rotation rate. For very high rotation rates, where the transitional region is close to the pole, the critical values of flow parameters for the onset of 
convective and absolute instabilities approach values consistent with those on the rotating disk. Physically this is of no surprise, as the sphere is locally flat near to the pole and so acts as a rotating disk in that region. For lower rotation rates, where the transitional behaviour manifests itself away from the pole, the effects of the sphere's surface curvature lead to distinct behaviour and critical parameters. The similarity between the rotating-disk flow and the flow around the sphere's pole can be shown mathematically using a simple series solution to approximate the steady-flow profiles in the boundary layer over the sphere, as first used by Banks [23]. Specifically, this approach demonstrates that the steady flow close to the pole is given by the von Kármán ordinary differential equations.

As demonstrated by Lingwood [1] and Garrett \& Peake [20], the absolute instability under consideration here exists as a result of inviscid effects. However, Healey [24] suggests pinch points resulting from a purely inviscid formulation (i.e. from the solution of the Rayleigh equation) are in fact distinct from those that exist in a viscous formulation (i.e. from the solution of an Orr-Sommerfeld-type equation). With this is in mind, we work with the viscous formulation throughout this paper.

\section{Formulation}

We consider a spherical body of revolution with surface described by the equation $r_{0}^{*}=r_{0}^{*}\left(s^{*}\right)$, where $s^{*}$ is the arc length measured along the surface of the body starting from the pole and $r_{0}^{*}\left(s^{*}\right)$ is the cross-sectional radius of the body in the plane perpendicular to the axis of symmetry. The body spins about its axis with angular velocity $\Omega^{*}$, in an otherwise undisturbed incom- 
pressible fluid. The asterisk denotes dimensional quantities. We consider typical length and time scales to be $\left(\nu^{*} / \Omega^{*}\right)^{1 / 2}$ and $\left(\nu^{*} / \Omega^{3}\right)^{1 / 2} / a^{*}$ respectively, where $\nu^{*}$ is the kinematic viscosity and $a^{*}$ is the sphere radius. We nondimensionalize $s^{*}$ and $r_{0}^{*}\left(s^{*}\right)$ with the typical length scale to form

$$
s=\frac{s^{*}}{\left(\nu^{*} / \Omega^{*}\right)^{1 / 2}}, \quad r_{0}(s)=\frac{r_{0}^{*}\left(s^{*}\right)}{\left(\nu^{*} / \Omega^{*}\right)^{1 / 2}},
$$

and define further nondimensional spatial variables

$$
\mathcal{S}=\frac{s^{*}}{a^{*}}, \quad R_{0}(\mathcal{S})=\frac{r_{0}^{*}}{a^{*}} .
$$

By eliminating $s^{*}$ and $r_{0}^{*}\left(s^{*}\right)$ between the two sets of scaled spatial variables we find that $\mathcal{S}$ is the slow spatial variable and $R_{0}$ is the slowly varying surface radius, i.e.

$$
\mathcal{S}=\epsilon s, \quad R_{0}(\mathcal{S})=\epsilon r_{0}
$$

with

$$
\epsilon=\frac{1}{a^{*}} \sqrt{\frac{\nu^{*}}{\Omega^{*}}},
$$

which is the ratio of the characteristic boundary-layer thickness to the characteristic size of the body. In what follows we assume that $\epsilon<<1$ which will be seen in Eq. (4) to be consistent with the assumption of large Reynolds number. From Barrow \& Garrett's [21] previous calculations, it follows that $\epsilon$ lies in the range $1 / 2883<\epsilon<1 / 240$, depending on the location at which local absolute instability is first observed between the pole and the equator respectively, which provides an a posteriori justification of the small $\epsilon$ analysis at each location over the sphere.

In this geometry it is clear that $R_{0}(\mathcal{S})=\sin \mathcal{S}$ and we see that the slow spatial variable $\mathcal{S}$ can be identified with the latitudinal angle, measured from the pole. 
We now introduce the transverse coordinate $\eta^{*} \equiv\left(\nu^{*} / \Omega^{*}\right)^{1 / 2} \eta$ which points in the normal direction out of the sphere, with $\eta=0$ being the sphere surface, together with the azimuthal angle $\phi$ measured around the axis. The coordinates $\mathcal{S}, \phi$ and $\eta$ form the coordinate system for our problem, and the fluid velocity has components $U^{*}, V^{*}, W^{*}$ in these respective directions. We write these velocity components in the form of an axisymmetric non-swirling steady flow plus an unsteady perturbation,

$$
\begin{aligned}
U^{*} & =a^{*} \Omega^{*}[U(\mathcal{S}, \eta)+\epsilon \bar{u}(\mathcal{S}, \phi, \eta, t)] \\
V^{*} & =a^{*} \Omega^{*}[V(\mathcal{S}, \eta)+\epsilon \bar{v}(\mathcal{S}, \phi, \eta, t)] \\
W^{*} & =a^{*} \Omega^{*}[\epsilon W(\mathcal{S}, \eta)+\epsilon \bar{w}(\mathcal{S}, \phi, \eta, t)],
\end{aligned}
$$

where $t$ is time nondimensionalized as indicated above, and the overbar denotes the unsteady perturbation. Note that the characteristic scale of the steady velocities in the $\mathcal{S}$ and $\phi$ directions is $a^{*} \Omega^{*}$, while the steady $\eta$ (wallnormal) velocity has scale $\left(\nu^{*} \Omega^{*}\right)^{1 / 2}$, which we have also taken as the scale of the unsteady perturbations. Finally, we note that the corresponding dimensional pressure can be written in the form

$$
\rho^{*}\left(a^{*} \Omega^{*}\right)^{2} \epsilon[\epsilon P(\mathcal{S}, \eta)+\bar{p}(\mathcal{S}, \phi, \eta, t)],
$$

where $\rho^{*}$ is the fluid density. These scalings define the Reynolds number of the system as

$$
R=\frac{a^{*} \Omega^{*} \sqrt{\nu^{*} / \Omega^{*}}}{\nu^{*}}=a^{*} \sqrt{\frac{\Omega^{*}}{\nu^{*}}}=\frac{1}{\epsilon} .
$$

The Reynolds number is therefore seen to be a direct measure of the scaled boundary-layer thickness and an indirect measure of the rotation rate, $\Omega^{*}$. This is in contrast to the interpretation of the Reynolds number in studies of 
the rotating disk, where, for fixed rotation rate, it gives the nondimensional radial position of the local stability analysis. For the sphere, the position of the local analysis is given by $\mathcal{S}$ for any rotation rate defined by $R$.

The equations for the steady boundary-layer flow around the sphere to leading order in $\epsilon$ are well known, see (2.2)-(2.4) of [20] after the substitution of $\mathcal{S}$ for $\theta$, for example. These partial differential equations in $\mathcal{S}$ and $\eta$ are solved subject to the no-slip and quiescent fluid boundary conditions. Further details of our numerical solution of these equations are given in that reference.

We now consider the unsteady flow where the resulting perturbation equations are given as (2.19)-(2.22) in [22] (after appropriate variable substitutions). In what follows we will be interested in the long-time response of the perturbation equations to initial forcing. Briggs and Bers $[9,10]$ showed that such behaviour can be analysed by investigating the dispersion properties of single-frequency homogeneous solutions. The Briggs-Bers procedure was developed for spatially homogeneous systems, but a significant extension was made by Monkewitz et al. [19], who considered weakly nonparallel flows which evolve only slowly in the streamwise direction. We therefore look for solutions of the perturbation equations of the form

$$
(\bar{u}, \bar{v}, \bar{w}, \bar{p})(\mathcal{S}, \eta, \phi, t)=(\tilde{u}, \tilde{v}, \tilde{w}, \tilde{p})(\mathcal{S}, \eta) \exp \left(\mathrm{i} n \phi-\mathrm{i} \gamma t+\frac{\mathrm{i}}{\epsilon} \int^{\mathcal{S}} \alpha\left(\mathcal{S}^{\prime}\right) \mathrm{d} \mathcal{S}^{\prime}\right) .
$$

Here $n$ must be an integer in order to enforce periodicity in the $\phi$ direction around the axis of symmetry. We will require $n$ to be large, and choose the preferred scaling $n=\bar{n} / \epsilon$, with $\bar{n}=O(1)$. It is crucial to note that we are looking for a global mode with azimuthal order which is the same at all $\mathcal{S}$. We now proceed by substituting (5) into the perturbation equations and, after completing a series of straightforward manipulations, we find the system 
identical to (2.13)-(2.18) in [20] after the simple substitution of $\beta=\bar{n} / \sin \mathcal{S}$ and $\theta=\mathcal{S}$. The resulting system of perturbation equations is listed in Appendix A for completeness.

As the steady flow is a mixed function of $\mathcal{S}$ and $\eta$, it is impossible to scale out $\bar{n}$ in the perturbation system and we must consider each value of $\bar{n}$ separately. The numerical solution of the perturbation system is completed in a standard fashion for each parameter pair of $R$ and $\bar{n}$ using a fourth-order Runge-Kutta integrator, starting from an analytical solution at the outer edge of the boundary layer (taken to be at $\eta=20$ ), and using a NewtonRaphson search procedure to solve the associated eigenvalue problem. Full details can be found in [22].

\section{Global modes}

Monkewitz et al. [19] show that the long-time linear behaviour of a weakly nonparallel flow is governed by the behaviour of the global mode of complex frequency $\gamma_{G}$ : If $\operatorname{Im}\left(\gamma_{G}\right)>0$ then the global mode is unstable, and hence the flow will be globally unstable, whereas if $\operatorname{Im}\left(\gamma_{G}\right)<0$ then the global mode is damped and the flow will be globally stable. The global-mode frequency is determined as follows. First, for each real $\mathcal{S}$ we look for a pinch in the complex $\alpha$ plane, i.e. for points of zero group velocity, $\partial \gamma / \partial \alpha=0$, formed by the coalescence of modes from opposite halves of the complex $\alpha$ plane. This provides us with a complex local absolute frequency, $\gamma=\gamma^{\circ}(\mathcal{S})$, along the real $\mathcal{S}$ axis. Second, we search for an $\mathcal{S}$ pinch point in $\gamma^{\circ}(\mathcal{S})$, which in general will occur at complex $\mathcal{S}$ and will therefore necessitate analytical continuation off the real $\mathcal{S}$ axis. In other words, we find a saddle point 
$\partial \gamma^{\circ} / \partial \mathcal{S}=0$, and then verify that the $\mathcal{S}$ contour can be deformed off the real axis so as to lie along the steepest descent contour through this saddle. Once these conditions have been satisfied, the global mode frequency simply corresponds to the frequency, $\gamma_{G}$, of this double $\alpha-\mathcal{S}$ pinch at the saddle location $\mathcal{S}_{S}$.

We solve the perturbation system (as given in Appendix A) for local absolute instability over the sphere by marching through the range of $\mathcal{S}$ in one degree increments for pairs of values of azimuthal wavenumber $\bar{n}$ and Reynolds number $R$. In practice, it is known that an eruption of the boundary layer occurs at the equator $\left(\mathcal{S}=90^{\circ}\right)$ and pollutes the steady flow around that region, and for this reason the study is confined to $\mathcal{S} \leq 80^{\circ}$. Typical results are demonstrated in Figure 1 where we show the absolute frequency $\gamma^{\circ}(\mathcal{S})$ for a sample of parameter pairs. Pockets of local absolute instability can be seen provided that $\bar{n}$ is sufficiently small and $R$ sufficiently large. Although not shown in Figure 1, our study considers all combinations of parameter pairs from $\bar{n}=0.05$ to 0.25 (in increments of 0.05 ) and $R=$ 100, 200, 300, 400, 500, 1000, 2000.

Unlike for the rotating disk/cone class of flows (see [25]), an analytical continuation of the absolute frequencies to complex $\mathcal{S}$ cannot be undertaken easily, due to the complicated dependence of the base flow on $\mathcal{S}$ in the governing partial differential equations. Instead we follow the suggestion of Cooper \& Crighton [26] and use Páde approximants. The idea is that a rational function is fitted to complex $\gamma^{\circ}(\mathcal{S})$ for real $\mathcal{S}$ (see [27], for example), which can then be interrogated to determine the location of any pinch point in complex $\mathcal{S}$ plane. After extensive tests with different orders of Páde approximant, it 
(a) $\gamma_{r}^{0}(\mathcal{S})$ at $R=300$.

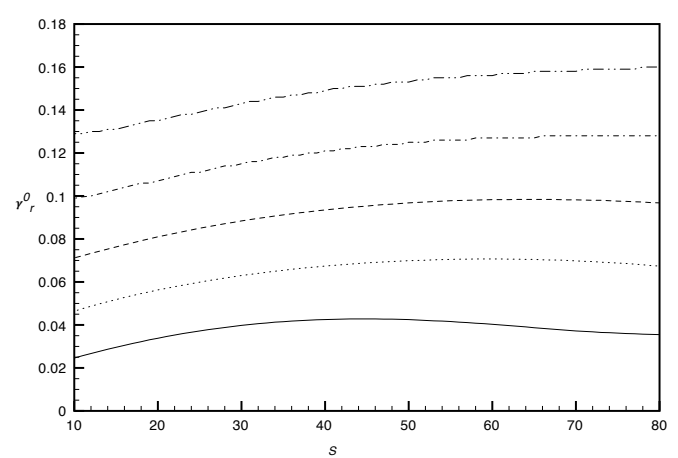

(c) $\gamma_{r}^{0}(\mathcal{S})$ at $R=500$.

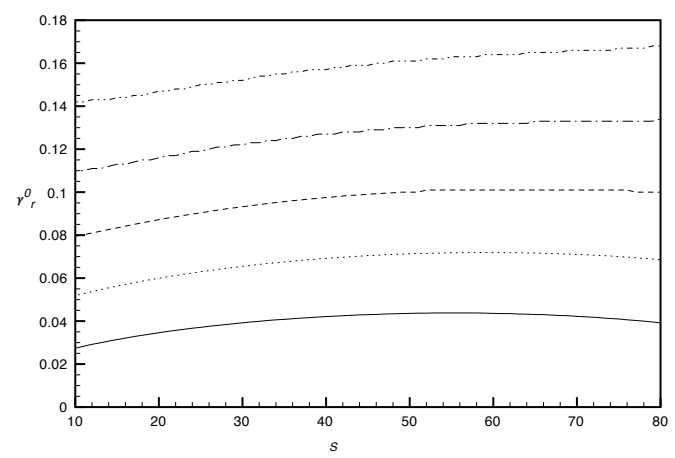

(e) $\gamma_{r}^{0}(\mathcal{S})$ at $R=1000$.

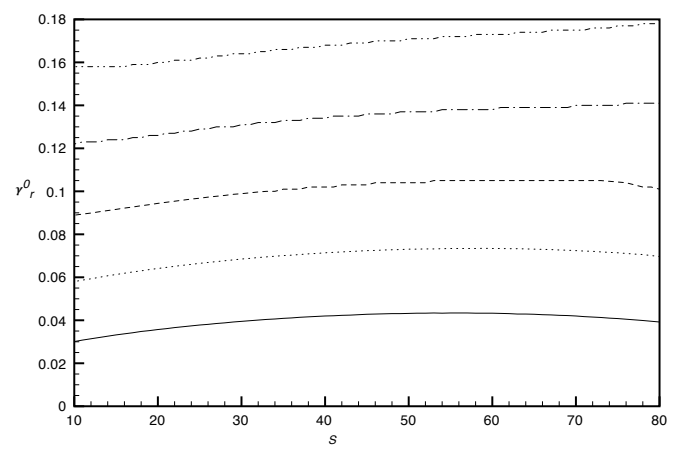

(b) $\gamma_{i}^{0}(\mathcal{S})$ at $R=300$.

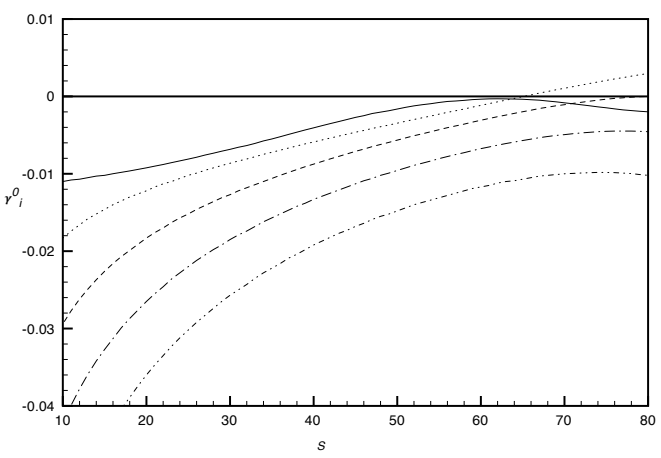

(d) $\gamma_{i}^{0}(\mathcal{S})$ at $R=500$.

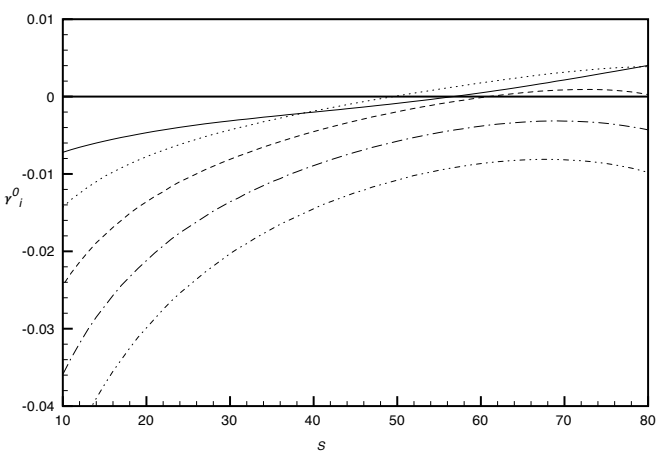

(f) $\gamma_{i}^{0}(\mathcal{S})$ at $R=1000$.

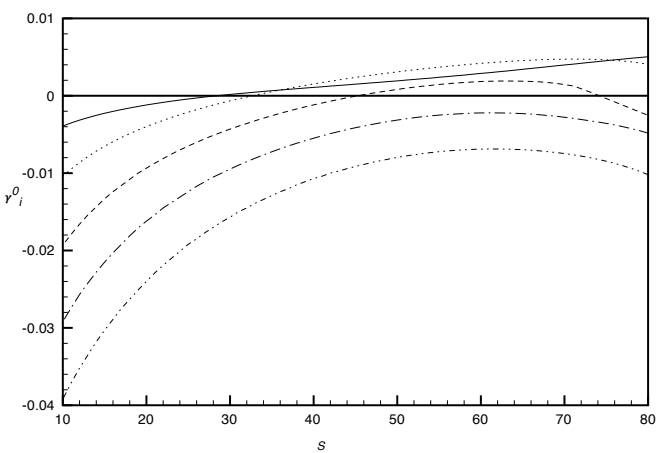

Figure 1: Plots of absolute frequency, $\gamma^{0}(\mathcal{S})$ at various $R$ for $\bar{n}=$ 0.05, 0.10, 0.15, 0.20, 0.25 “_", “...”, “- -", “-.”, “-..”, respectively. 
was found that using polynomials of order five for both the numerator and the denominator typically gave the smallest error for each parameter set. In Cooper \& Crighton's notation, $E_{m}$, the root mean square error incurred by using the Páde function to approximate the data on the real axis, is at worst $O\left(10^{-4}\right)$. This method of approximation yields a complex absolute frequency which, for real $\mathcal{S}$, agrees with the original results to three decimal places for all parameter values considered here.

Figure 2 shows sample contours of $\gamma_{i}^{\circ}$ in the complex $\mathcal{S}$ plane for a small sample of $\bar{n}, R$ parameter pairs. Saddle points are visible towards the right of each plot and are marked with a '*'. The thicker lines represent the zero contour $\left(\gamma_{i}^{\circ}=0\right)$. Table 1 gives the positions of the saddle points, $\mathcal{S}_{S}$, and the associated global frequencies, $\gamma_{G}$, for a range of parameters.

We are able to plot the neutral curve for linear global instability by repeating the analysis at various $\bar{n}$ and recording $R$ such that $\operatorname{Im}\left(\gamma_{G}\right)=0$; this is shown in Figure 3.

The results in Table 1 and Figure 3 show that a linear global mode exists in the boundary-layer flow over the rotating sphere. The mode is damped for rotation rates corresponding to $R$ below a critical value of $R_{\text {crit }}=337$ (which occurs at $\bar{n}=0.11)$. As the rotation rate is increased beyond this, the range of $\bar{n}$ for which a self-sustained global mode can exist broadens, reflecting the increased extent of the pockets of absolute instabilities that exist at these parameter values. Interestingly, Table 1 shows that the properties of the unstable global mode at each $R$ appear to be fixed by properties of the flow at latitudes between approximately $55^{\circ}-65^{\circ}$ for all $R \leq 2000$, by which point the boundary layer is known to be locally absolutely unstable 

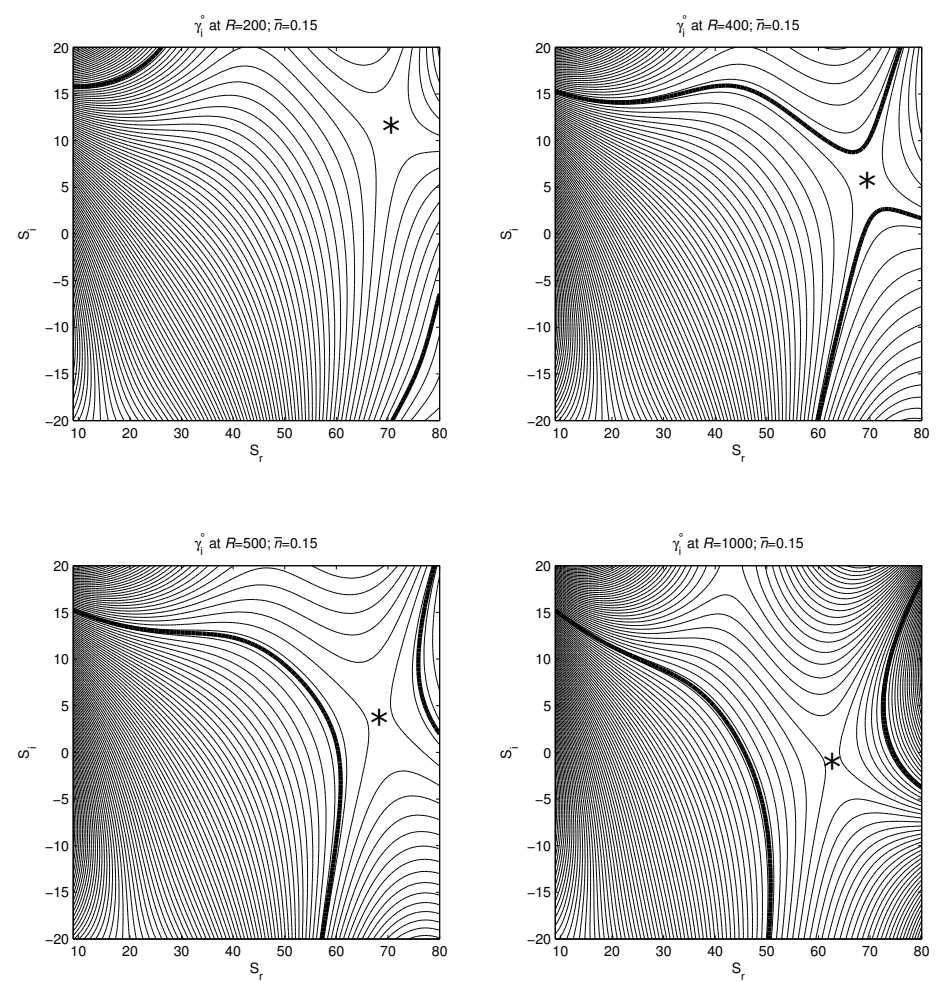

Figure 2: Level curves of $\gamma_{i}^{\circ}$ in the complex $\mathcal{S}$ plane at $\bar{n}=0.15$. Saddle points are marked with '*'. Here $\mathcal{S}$ is measured in degrees. 


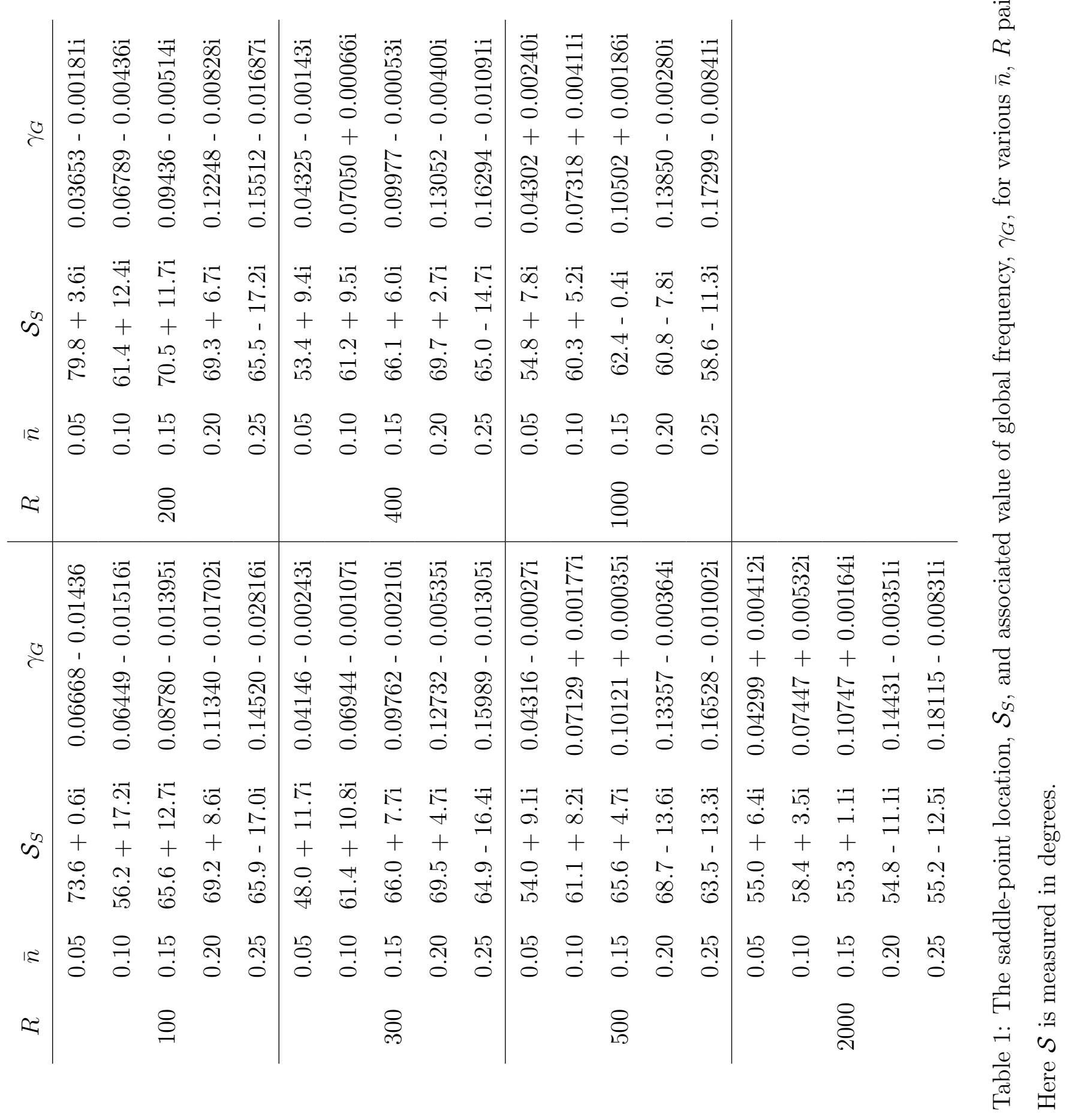




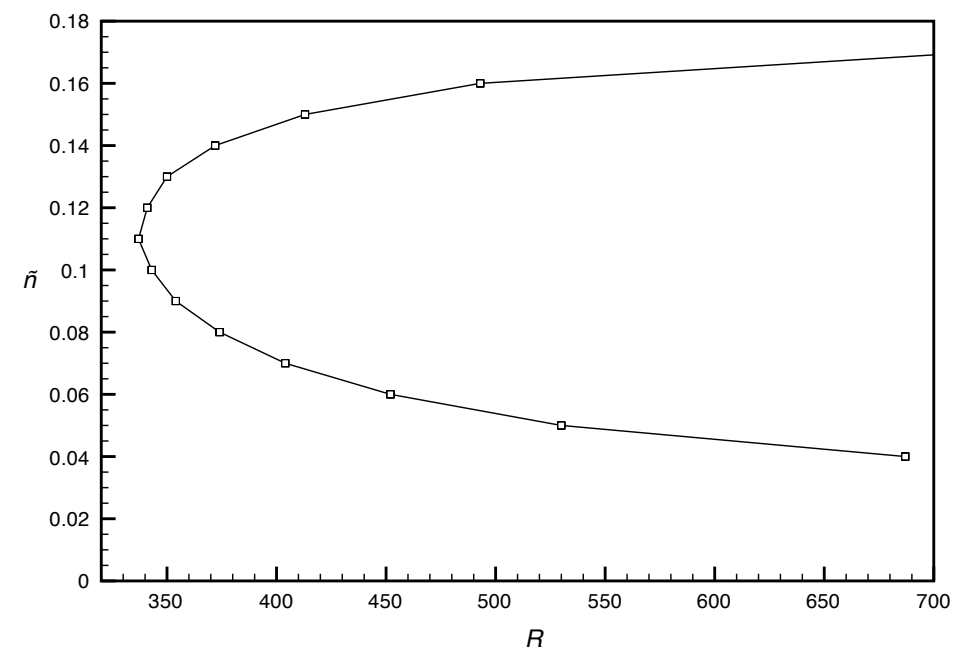

Figure 3: Neutral curve for the onset of linear global instability.

at all latitudes above approximately $15^{\circ}$ (see $[20,21]$ ). It is worth noting that these critical latitudes are well away from the high latitude where the boundary layer solution is terminated. Also note, from Table 1 , that the $\mathcal{S}$ saddles are relatively close to the real $\mathcal{S}$ axis, which suggests that the analytic continuation is reliable.

It is interesting to compare the theoretical onset of local absolute instability [20, 21] and experimental measurements for the onset of turbulence $[28,29]$ at each latitude with our predicted onset of the linear global mode. This comparison is presented in Figure 4 in terms of the spin Reynolds number $\left(R_{S}=R^{2}\right)$ that has been used to report experimental results in the literature. The figure shows the onset of local absolute instability as a function of latitude, $\mathcal{S}$, to be roughly parallel to the observed onset of turbulence, although we see an increasing discrepancy as the analysis moves to higher $\mathcal{S}$. The experimental results appear to tend to a particular value of spin 


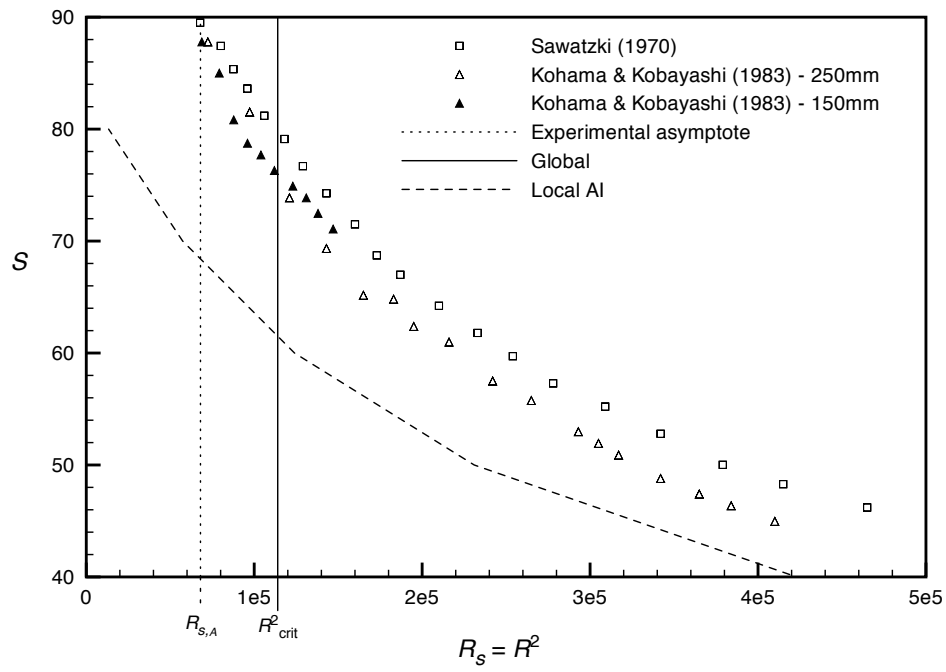

Figure 4: Theoretical onset of local absolute instability, the unstable linear global mode and experimental data for the onset of turbulence [28, 29].

Reynolds number, denoted $R_{S, A}$, as $\mathcal{S}$ tends to the equator. This limiting value represents the minimum Reynolds number that is required to observe turbulence on spheres of any radius, and can be used to calculate the minimum spin rate required to observe turbulence close to the equator of a sphere of particular radius. One might expect $R_{S, A}$ to be associated with $R_{\text {crit }}$ and the two values are indeed reasonably consistent. As discussed by Lingwood [2] for example, the measurement of the precise onset of turbulence is open to some experimental discretion, and this, together with the inaccuracies arising from the Páde approximation used in this analysis, could partly explain the discrepancy between the values of $R_{S, A}$ and $R_{\text {crit }}$. Furthermore, the limitations of a linear theory as compared to measurements of a nonlinear physical system are bound to manifest at this stage. 


\section{Conclusions}

Our results suggest that the boundary-layer flow over a sphere rotating in an otherwise still fluid can support self-sustained linear global modes if it is rotated sufficiently fast. This conclusion has been reached with knowledge of the local absolute instability properties of the boundary layer [20, 21] which we have used to locate the complex $\mathcal{S}$ saddle point. The critical Reynolds number for the onset of the linear global mode, $R_{\text {crit }}=337$, is reasonably consistent with the observed minimum Reynolds number for the appearance of turbulence in experimental studies and the discrepancy could be due to the inaccuracies arising within both the experimental and theoretical studies.

The existence of the unstable linear global mode is in contrast to the literature concerning the linear global modes over a rotating disk [3, 5], where it is generally accepted that self-sustained linear global modes do not exist. It is important to note that our results do not contradict this result as the unstable linear global mode we have found on the sphere appears to be fixed by properties of the flow at latitudes between $55^{\circ}-65^{\circ}$. This location is well away from the pole where the boundary-layer flow over the sphere approximates that of the disk. We have no evidence to suggest that further increases in Reynolds number would lead to the global properties of the flow being fixed closer to the pole. Our results suggest that, despite both being susceptible to local absolute instability, the mechanisms by which transition to turbulence occurs over rotating disks and spheres could be fundamentally different. The next stage in this continuing work is to study the effects of increased rotation rate and the connection between the properties of the rotating sphere near to the pole and the rotating disk. 
The authors are delighted to dedicate this work to Professor Patrick Huerre, and join in recognising his enormous and seminal contributions to the theory of hydrodynamic stability.

This work was supported by the Engineering and Physical Sciences Research Council (EP/G061637/1) and the University of Leicester. SJG wishes to acknowledge study leave from the University of Leicester and the kind hospitality of KTH and NORDITA, Stockholm, where this paper was drafted.

\section{A. Governing perturbation equations}

The perturbation equations can be written as a set of six first-order ordinary differential equations using the transformed dependent variables

$$
\begin{aligned}
& z_{1}(\eta ; \alpha, \bar{n}, \gamma ; R, \mathcal{S})=(\alpha-\mathrm{i} \cot \mathcal{S} / R) u+(\bar{n} / \sin \mathcal{S}) v \\
& z_{2}(\eta ; \alpha, \bar{n}, \gamma ; R, \mathcal{S})=(\alpha-\mathrm{i} \cot \mathcal{S} / R) D u+(\bar{n} / \sin \mathcal{S}) D v \\
& z_{3}(\eta ; \alpha, \bar{n}, \gamma ; R, \mathcal{S})=w \\
& z_{4}(\eta ; \alpha, \bar{n}, \gamma ; R, \mathcal{S})=p \\
& z_{5}(\eta ; \alpha, \bar{n}, \gamma ; R, \mathcal{S})=(\alpha-\mathrm{i} \cot \mathcal{S} / R) v-(\bar{n} / \sin \mathcal{S}) u \\
& z_{6}(\eta ; \alpha, \bar{n}, \gamma ; R, \mathcal{S})=(\alpha-\mathrm{i} \cot \mathcal{S} / R) D v-(\bar{n} / \sin \mathcal{S}) D u
\end{aligned}
$$

where $D$ represents differentiation with respect to $\eta$. Writing $\alpha_{1}=\alpha-$ $[\mathrm{i} \cot \mathcal{S} / R]_{s}$, these equations are 


$$
\begin{aligned}
& D z_{1}=z_{2}, \\
& {\left[\frac{D z_{2}}{R}\right]_{v}=\frac{1}{R}\left(\left[\alpha^{2}+\left(\frac{\bar{n}}{\sin \mathcal{S}}\right)^{2}\right]_{v}+\mathrm{i} R\left(\alpha U+\frac{\bar{n}}{\sin \mathcal{S}} V-\gamma\right)\right) z_{1}} \\
& +\left[\frac{W z_{2}}{R}\right]_{s}+\left(\alpha_{1} U^{\prime}+\frac{\bar{n}}{\sin \mathcal{S}} V^{\prime}+\left[\frac{1}{R}\left(\alpha_{1} U+\frac{\bar{n}}{\sin \mathcal{S}} V\right)\right]_{s}\right) z_{3} \\
& +\mathrm{i}\left(\alpha^{2}+\left(\frac{\bar{n}}{\sin \mathcal{S}}\right)^{2}-\left[\frac{\mathrm{i} \alpha \cot \mathcal{S}}{R}\right]_{s}\right) z_{4}-\left[\frac{V \cot \mathcal{S} z_{5}}{R}\right]_{s} \\
& +\left[\frac{1}{R}\left(\left(\alpha_{1} \frac{\partial U}{\partial \mathcal{S}}+\frac{\bar{n}}{\sin \mathcal{S}} \frac{\partial V}{\partial \mathcal{S}}\right) u-\left(\alpha_{1} V-\frac{\bar{n}}{\sin \mathcal{S}} U\right) v \cot \mathcal{S}\right)\right]_{s}, \\
& D z_{3}=-\mathrm{i} \phi_{1}-\left[\frac{2 z_{3}}{R}\right]_{s}, \\
& D z_{4}=\left[\frac{\mathrm{i} W z_{1}}{R}\right]_{s}-\left[\frac{\mathrm{i} z_{2}}{R}\right]_{v}+\left[\frac{2}{R}(U u+V v)\right]_{s} \\
& -\frac{1}{R}\left(\left[\alpha^{2}+\left(\frac{\bar{n}}{\sin \mathcal{S}}\right)^{2}\right]_{v}+\mathrm{i} R\left(\alpha U+\frac{\bar{n}}{\sin \mathcal{S}} V-\gamma\right)+D W_{s}\right) z_{3}, \\
& D z_{5}=z_{6}, \\
& {\left[\frac{D z_{6}}{R}\right]_{v}=\left[\frac{V \cot \mathcal{S} z_{1}}{R}\right]_{s}+\left(\alpha_{1} \frac{\partial V}{\partial \eta}-\frac{\bar{n}}{\sin \mathcal{S}} \frac{\partial U}{\partial \eta}+\left[\frac{1}{R}\left(\alpha_{1} V-\frac{\bar{n}}{\sin \mathcal{S}} U\right)\right]_{s}\right) z_{3}} \\
& +\left[\frac{W z_{6}}{R}\right]_{s}+\left[\frac{1}{R}\left(\left(\alpha_{1} \frac{\partial V}{\partial \mathcal{S}}-\frac{\bar{n}}{\sin \mathcal{S}} \frac{\partial U}{\partial \mathcal{S}}\right) u+\left(\alpha_{1} U+\frac{\bar{n}}{\sin \mathcal{S}} V\right) v \cot \mathcal{S}\right)\right]_{s} \\
& +\left[\frac{\frac{\bar{n}}{\sin \mathcal{S}} \cot \mathcal{S} z_{4}}{R}\right]_{s}+\frac{1}{R}\left(\left[\alpha^{2}+\left(\frac{\bar{n}}{\sin \mathcal{S}}\right)^{2}\right]_{v}+\mathrm{i} R\left(\alpha U+\frac{\bar{n}}{\sin \mathcal{S}} V-\gamma\right)\right) z_{5} .
\end{aligned}
$$

The subscripts $v$ and $s$ indicate which of the $O\left(R^{-1}\right)$ terms arise from the viscous and streamline-curvature effects respectively. Note that since a stationary frame of reference is used Coriolis terms do not appear in the governing equations). Note also that the perturbation velocities $u$ and $v$ still 
appear explicitly, but can be expressed in terms of $z_{1}$ and $z_{2}$ via

$$
\begin{aligned}
& u=\frac{1}{\alpha_{1}^{2}+(\bar{n} / \sin \mathcal{S})^{2}}\left(\alpha_{1} z_{1}-\frac{\bar{n}}{\sin \mathcal{S}} z_{5}\right), \\
& v=\frac{1}{\alpha_{1}^{2}+(\bar{n} / \sin \mathcal{S})^{2}}\left(\alpha_{1} z_{5}+\frac{\bar{n}}{\sin \mathcal{S}} z_{1}\right) .
\end{aligned}
$$

\section{References}

[1] Lingwood, R.J. 1995 Absolute instability of the boundary layer on a rotating disk. J. Fluid Mech., 299, 17-33.

[2] Lingwood, R.J. 1996 An experimental study of absolute instability of the rotating-disk boundary-layer flow. J. Fluid Mech., 314, 405-428.

[3] Davies, C.\& Carpenter, P.W. 2003 Global behaviour corresponding to the absolute instability of the rotating-disk boundary layer. $J$. Fluid Mech., 486, 287-329.

[4] Othman, H. \& Corke, T.C. 2006 Experimental investigation of absolute instability of a rotating-disk boundary layer. J. Fluid Mech, 565, 63-94.

[5] PIER, B. 2003 Finite-amplitude crossflow vortices, secondary instability and transition in the rotating-disk boundary layer. J. Fluid Mech, 487, $315-343$.

[6] Huerre, P. \& Monkewitz, P.A. 1990 Local and global instabilities in spatially developing flows. Annu. Rev. Fluid Mech., 22, 473-537.

[7] BAnks, W.H.H. 1976 The boundary layer on a rotating sphere. Q. J. Mech. Appl. Math., 18, 443-454. 
[8] Barrow, A. \& Garrett, S.J. 2013 The stability and transition of the boundary-layer flow over rotating spheres with surface mass flux and incident axial flow, Eur. J. Mech. B., 38, 93-100.

[9] BRIgGs, R.J. 1964 Electron-stream Interaction with Plasmas . Monograph No. 29. MIT Press.

[10] Bers, A. 1983 Space-time evolution of plasma instabilities - absolute and convective. In Handbook of Plasma Physics, ed M.N. Rosenbluth, R. Z. Sagdeev. 1:451-517. North-Holland.

[11] Kohama, Y. 1984 Study on boundary layer transition of a rotating disk. Acta Mech., 50, 193-199.

[12] Imayama, S., Alfredsson, P.H. \& Lingwood, R.J. 2012 A new way to describe the transition characteristics of a rotating-disk boundary-layer flow. Phys. Fluids, 24, 031701.

[13] Siddiqui, M.E., Mukund, V., Scott, J. \& Pier, B. 2013 Experimental characterization of transition region in rotating-disk boundary layer. Phys. Fluids, 25, 034102.

[14] Viaud, B., Serre, E. \& Chomaz, J-M 2008 The elephant mode between two rotating disks. J. Fluid Mech, 598, 451-464.

[15] Viaud, B., Serre, E. \& Chomaz, J-M 2011 Transition to turbulence through steep global-mode cascade in an open rotating cavity. J. Fluid Mech, 688, 493-506. 
[16] Healey, J.J. 2010 Model for unstable global modes in the rotating-disk boundary layer. J. Fluid Mech., 663, 148-159.

[17] Imayama, S., Alfredsson, P.H. \& Lingwood, R.J. 2013 An experimental study of edge effects on rotating-disk transition. J. Fluid Mech., 716, 638-657.

[18] PIER, B. 2013 Transition near the edge of a rotating disk. J. Fluid Mech., 737, R1.

[19] Monkewitz, P.A., Huerre, P. \& Chomaz, J-M 1993 Global linear stability analysis of weakly non-parallel shear flows. J. Fluid Mech., 251, $1-20$.

[20] Garrett, S.J.\& Peake, N. 2002 The stability and transition of the boundary layer on a rotating sphere. J. Fluid Mech., 456, 199-218.

[21] Barrow, A. \& Garrett, S.J. 2013 The stability and transition of the boundary-layer flow over rotating spheres with surface mass flux and incident axial flow. European J. Mech. B., 38, 93-100.

[22] Garrett, S.J. 2002 The stability and transition of the boundary layer on rotating bodies. PhD Thesis, University of Cambridge.

[23] BANKs, W.H.H. 1976, Laminar boundary-layer on a rotating sphere, Acta Mech., 24, 273-287.

[24] Healey, J.J. 2004 On the relation between the viscous and inviscid absolute instabilities of the rotating-disk boundary layer. J. Fluid Mech., 511, 179-199. 
[25] Garrett, S.J.\& Peake, N. 2007 On the global linear stability of the boundary layer on rotating bodies. Advances in Turbulence XI: Proceedings of the 11th EUROMECH European Turbulence Conference, ed. J.M.L.M Palma, A. Silva Lopes, 550-552.

[26] Cooper, A. J. \& Crighton, D. G. 2000 Global modes and superdirective acoustic radiation in low-speed axisymmetric jets. Eur. J. Mech. B - Fluids 19, 559-574.

[27] Press, W.H., Teukolsky, S.A., Vetterling, W.T. \& FlanNERY, B.P. 1992 Numerical Recipes in Fortran. Cambridge.

[28] Sawatzki, O. 1970 Das Strömungsfeld um eine rotierende Kugel. Acta Mech. 9, 159-214.

[29] Kohama, Y. \& Kobayashi, R. 1983 Boundary-layer transition and the behaviour of spiral vortices on rotating spheres. J. Fluid Mech. 137, 153-164. 